\title{
Multiparametric scaling relations for dwarf irregular galaxies in different environments
}

\author{
M. E. Sharina, ${ }^{1}$ V. E. Karachentseva, ${ }^{2}$ and D. I. Makarov ${ }^{1}$ \\ ${ }^{1}$ Special Astrophysical Observatory, Russian Academy of Sciences, N. Arkhyz, KChR, 369167, \\ Russia \\ email: sme@sao.ru \\ ${ }^{2}$ Main Astronomical Observatory, National Academy of Sciences of Ukraine, Ukraine \\ email: valkarach@gmail.com
}

\begin{abstract}
We study the correlations of rotation velocity and absolute magnitude with surface brightness for low surface brightness dwarf irregular galaxies (dIrrs). We find that isolated objects contribute most to the scatter in the Tully-Fisher relation (TFR). Excluding these extreme cases, we develop a three-parameter (luminosity, Hi line width at $20 \%$ of peak flux level, i.e., $W_{20}$, effective surface brightness) TFR for 60 dIrrs (with revised Hubble type $T>8$ ) in the Local Volume (LV) with Cepheid and tip-of-the-red-giant-branch distance measurements. The relation is applied to galaxies of the same morphological type with radial velocities $v_{\mathrm{LG}} \leqslant 3500$ $\mathrm{km} \mathrm{s}^{-1}$ in the Local Supercluster. We obtained surface photometry and determined structural parameters using sDss images. The rotational velocities and derived photometric parameters for most galaxies in small groups agree well with those corresponding to the three-parameter TFR. However, isolated galaxies appear to have systematically lower surface brightnesses and longer scale lengths for the same luminosity than galaxies in small groups. This may indicate on average twice larger HI-to-optical disk size ratios for our sample of isolated dIrrs, because their Hi surface densities calculated using the optical diameters look normal.
\end{abstract}

Keywords. galaxies: distances and redshifts, galaxies: fundamental parameters, galaxies: dwarf, galaxies: irregular

\section{On the physical scatter in the Tully-Fisher relation}

The Tully-Fisher relation (TFR; Tully \& Fisher 1977) implies that the dark matter and baryonic content of regularly rotating disk-like galaxies are tightly correlated (e.g., McGaugh 2005). The scatter in the TFR for bright spiral galaxies is small. The TFR is wavelength-dependent (Aaronson \& Mould 1983; Sakai et al. 2000) and it differs for field and cluster galaxies (Sandage \& Tammann 1984). Dwarf galaxies have shallow potential wells. They lose baryons due to bursts of star formation and supernova winds, and often fall below the TFR defined by bright galaxies (e.g., Efstathiou 2000; Begum et al. 2008; McGaugh et al. 2010). Variations in mass-to-light $(M / L)$ ratios, gas fractions, and rotation velocities at a given mass for different galaxies are sources of physical scatter in the TFR (Geha et al. 2006; Kannappan et al. 2002).

dIrrs have similar colors (see Table 1 ), so their stellar $M / L$ ratios are expected to be similar (Bell \& de Jong 2001). Uncertainties due to internal interstellar extinction for dIrrs are small (James et al. 2005). However, variations in their structure, surface brightness, gas fraction, and rotation curve asymmetry are considerable. The scatter in the TFR is small in the infrared (e.g., McCall et al. 2012; Sorce et al. 2012). A comparative study of the TFR in optical bands and of its correlation with different distance-independent galactic properties will help to solve galaxy formation problems (e.g., Eisenstein \& Loeb 1996; Kannappan et al. 2002). 
Table 1. Mean photometric properties of dwarf spheroidal, irregular, and transition-type galaxies in the Local Group and the Local Volume: broad-band $(V-I),(B-V)$ colors, central surface brightnesses, and absolute $V$-band magnitudes. All data have been corrected for Galactic foreground extinction.

\begin{tabular}{lcccccccc}
\hline Morph. Type & $(V-I)_{\mathrm{LG}}$ & $(V-I)_{\mathrm{LV}}$ & $(B-V)_{\mathrm{LG}}$ & $(B-V)_{\mathrm{LV}}$ & $\mathrm{SB}_{V, \mathrm{LG}}$ & $\mathrm{SB}_{V, \mathrm{LV}}$ & $M_{V, \mathrm{LG}}$ & $M_{V, \mathrm{LV}}$ \\
\hline $\mathrm{dSph}$ & $1.20 \pm 0.20$ & $1.10 \pm 0.12$ & $0.70 \pm 0.10$ & $0.80 \pm 0.20$ & 24.61 & 23.36 & -11.31 & -11.07 \\
$\mathrm{dIrr} / \mathrm{dSph}$ & $1.00 \pm 0.10$ & $0.90 \pm 0.12$ & $0.68 \pm 0.10$ & $0.57 \pm 0.10$ & 23.90 & 22.75 & -10.86 & -12.79 \\
$\mathrm{dIrr}$ & $0.73 \pm 0.20$ & $0.74 \pm 0.20$ & $0.46 \pm 0.10$ & $0.48 \pm 0.20$ & 22.43 & 22.36 & -12.89 & -12.97 \\
\hline
\end{tabular}

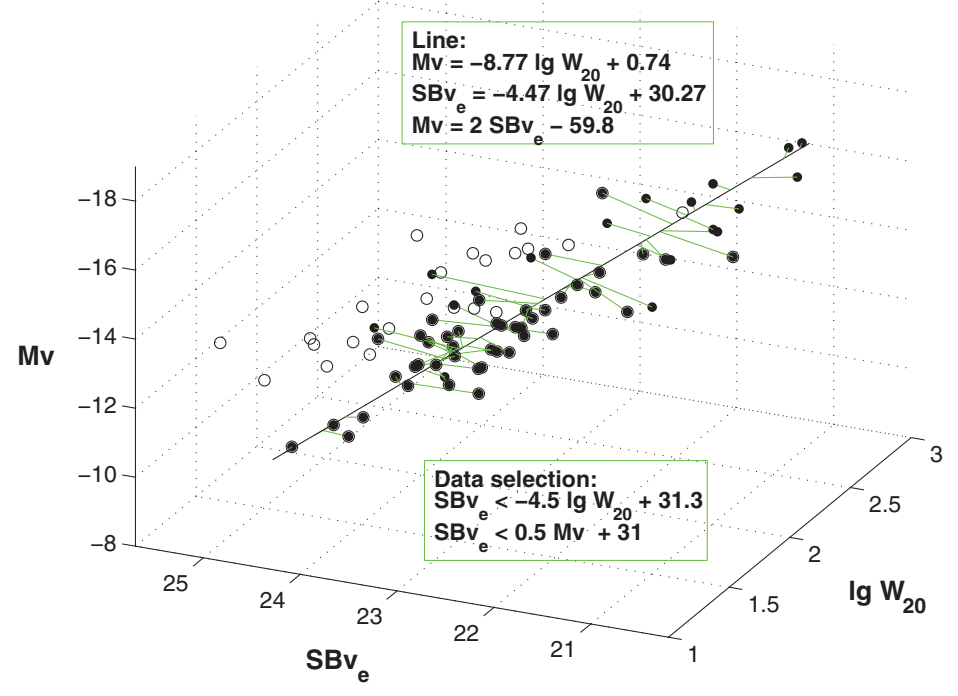

Figure 1. Fundamental plane for irregular galaxies in the Local Volume (LV); dIrrs with deviations of more than $3 \sigma$ from the three-parameter TFR are shown as open circles.

Our sample includes dIrrs in the Local Supercluster with $v_{\mathrm{LG}} \leqslant 3500 \mathrm{~km} \mathrm{~s}^{-1}$ that are isolated (Karachentseva et al. 2010, hereafter KKS10) and located in the region defined by R.A., Dec $(J 2000.0)=\left[10-12^{\mathrm{h}}\right],\left[-5-+35^{\circ}\right]$. Galaxies around the Virgo Cluster are influenced by its gravitational field. It is important to know the environmental status of these objects. Therefore, we use the so-called 'isolation index' of Makarov \& Karachentsev (2011). Half of our objects belong to the Leo I and Leo II groups. The other half reside in smaller groups.

\section{The three-parameter Tully-Fisher relation}

The 3D TFR obtained using principal component analysis for dIrrs in the LV (Karachentsev et al. 2004, hereafter CNG04) is shown in Fig. 1. Photometric data were taken from Sharina et al. (2008) and from the literature. We considered the mean surface brightness within the effective radius in the $V$ and $I$ bands and the $\mathrm{HI}$ line width at $20 \%$ of the peak flux level $\left(W_{20}\right)$, following Begum et al. (2008). Three equations for the best-fitting line and the data selection conditions are included in the figure.

The $\lg W_{20}-\mathrm{SB}_{I, \mathrm{e}}$ projection of the plane is demonstrated in Fig. 2a. It is seen that there are deviations from the best-fitting TFR and that they are populated mostly by isolated galaxies. Their tidal indices (CNG04), indicated in the figure, are negative. 

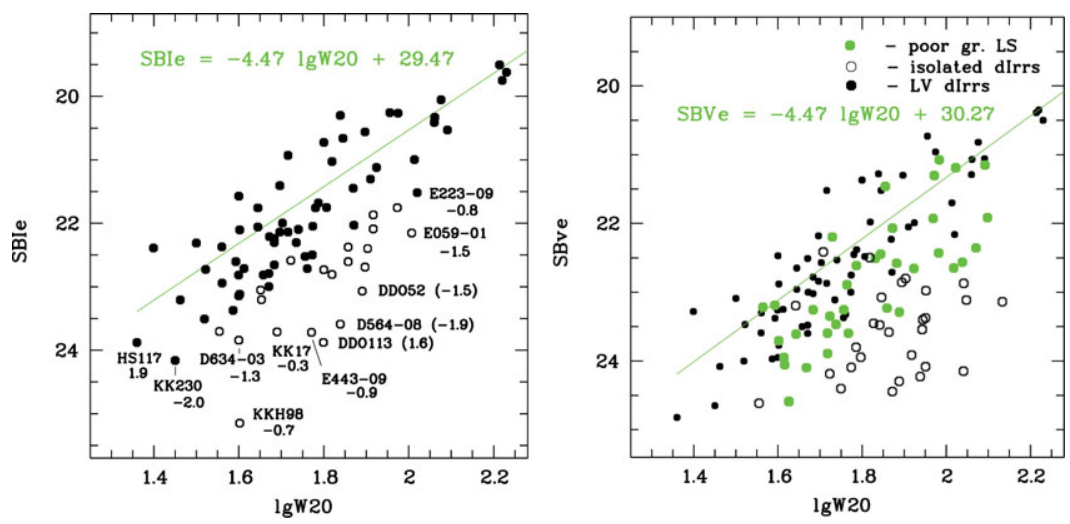

Figure 2. (a) $\mathrm{SB}_{I, \mathrm{e}}$ versus $\lg W_{20}$ for dIrrs in the LV. Symbols are the same as in Fig.1. Tidal indices are indicated for some galaxies with surface brightnesses that are too low at a given $\lg W_{20}$. (b) $\mathrm{SB}_{V, \text { e }}$ versus $\lg W_{20}$ for all dIrrs in our sample. Projections of the TFR (Fig. 1 ) in the $V$ and $I$ bands are overplotted. LS: Local Supercluster.

Fig. 2b shows that our sample dIrrs in small groups around the Virgo Cluster follow the best-fitting TFR. However, isolated dIrrs from KKS10 deviate systematically towards lower surface brightness.

\section{HI surface densities}

Isolated dIrrs have $\sim 2.5 \times$ enhanced median Hi content compared with non-isolated dIrr galaxies (Karachentseva et al. 2010). CNG04 detected a 30\% decrease in Hi surface brightness in the most isolated galaxies with respect to the most interacting (disturbed) dIrrs. The median Hi surface density is $\sim 10^{7} \mathrm{M}_{\odot} \mathrm{kpc}^{-2}$ for disk-like galaxies in the LV (CNG04). Fig. 3 demonstrates the relation between $V$-band surface brightness and $\mathrm{HI}$ surface density for our sample objects. We do not see any significant differences between the distributions of dIrrs in different environments. There is a concentration of galaxies at $\mathrm{SB}_{\mathrm{HI}} \sim 10^{7} \mathrm{M}_{\odot} \mathrm{kpc}^{-2}$ and $\mathrm{SB}_{V, \mathrm{e}} \sim 23.4 \mathrm{mag} \operatorname{arcsec}^{-2}$ and a slight increase of $\mathrm{SB}_{V, \mathrm{e}}$ with increasing $\lg \mathrm{SB}_{\mathrm{HI}}$. Relatively empty regions in the diagram highlighted by triangles include exotic objects: bright starburst galaxies with active star formation and relatively low Hi surface densities (IC 5152, NGC 4605, NGC 1156), or instead low surface brightness objects with enormous HI reservoirs (DDO 13, ESO 379-07, DDO 154).

\section{Conclusions and future work}

We developed an optical three-parameter TFR for dIrrs in the LV. Our aim is to show that any deviations from this relationship are caused by different HI-to-optical disk size ratios for the sample galaxies. We will continue to work on deriving new and more accurate distances to our sample galaxies based on both optical photometric and HI data. This will help to improve our knowledge of the physical sources of the scatter in the TFR.

\section{Acknowledgements}

We thank the Symposium organizers for putting together this very interesting meeting and the editor Richard de Grijs. We acknowledge support from the Ministry of Education and Science of the Russian Federation, under contract 14.740.11.0901 and proposal 2012-1.5-12-000-1011-004. We acknowledge use of the HYPERLEDA database (http://leda.univ- 

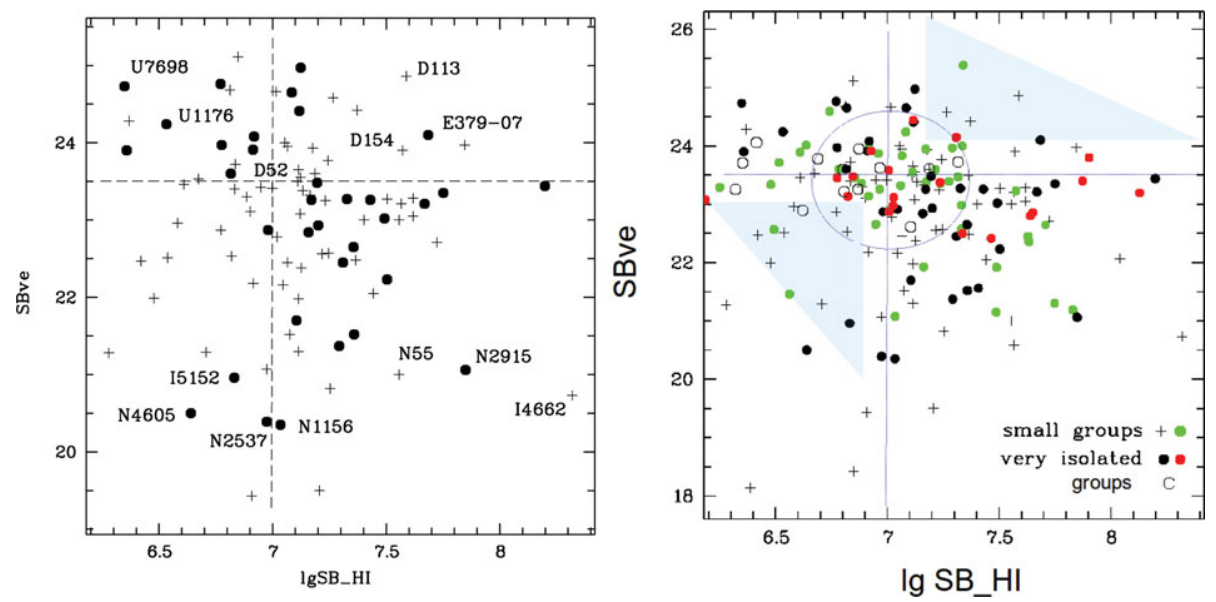

Figure 3. Hi surface brightness $\left(\mathrm{M}_{\odot} \mathrm{kpc}^{-2}\right)$ within the optical diameter versus $\mathrm{SB}_{V, \mathrm{e}}$ for (left) the LV and (right) all galaxies in this study. Very isolated galaxies with tidal indices $<-1$ are shown as black dots. The concentration of galaxies near $\lg \mathrm{SB}_{\mathrm{HI}} \sim 10^{7} \mathrm{M}_{\odot} \mathrm{kpc}^{-2}$, $\mathrm{SB}_{V, \mathrm{e}} \sim 23.4 \mathrm{mag}$ arcsec ${ }^{-2}$ is indicated by a large circle. Relatively empty regions in the diagram are highlighted as transparent triangles.

lyon1.fr). Funding for the SDSS and SDSS-II has been provided by the Alfred P. Sloan Foundation, the participating institutions, the U. S. National Science Foundation, the U. S. Department of Energy, the National Aeronautics and Space Administration, the Japanese Monbukagakusho, the Max Planck Society, and the Higher Education Funding Council for England. The sDss Website is http://www.sdss.org.

\section{References}

Aaronson, M. \& Mould, J. 1983, ApJ, 265, 1

Begum A., Chengalur, J. N., Karachentsev, I. D., \& Sharina, M. E. 2008, MNRAS, 386, 138

Bell, E. F. \& de Jong, R. S. 2001, ApJ, 550, 212

Efstathiou, G. 2000, MNRAS, 317, 697

Eisenstein, D. J. \& Loeb, A. 1996, ApJ, 459, 432

Geha, M., Blanton, M. R., Masjedi, M., \& West, A. A. 2006, ApJ, 653, 240

James, P. A., Shane, N. S., Knapen, J. H., Etherton, J., \& Percival, S. M., 2005, A\&A, 429, 851

Kannappan, S. J., Fabricant, D. G., \& Franx, M. 2002, AJ, 123, 2358

Makarov, D. I. \& Karachentsev, I. D. 2011, MNRAS, 412, 2498

Karachentsev, I. D., Karachentseva, V. E., Huchtmeier, W. K., \& Makarov, D. I. 2004, AJ, 127, 2031 (CNG04)

Karachentseva, V. E., Karachentsev, I. D., \& Sharina, M. E. 2010, Astrophys., 53, 462 (arXiv: 1104.2506; KKS10)

McCall M. L., Vaduvescu O., Pozo Nunez F., et al. 2012, A\& A, 540, 49

McGaugh, S. S., Schombert, J. M., de Blok, W. J. G., \& Zagursky, M. J. 2010, ApJ, 708, L14

McGaugh, S. S. 2005, ApJ, 632, 859

Sakai, S., Mould, J. R., Hughes, S. M. G., et al. 2010, ApJ, 529, 698

Sandage, A. \& Tammann, G. 1984, Nature, 307, 326

Sharina, M. E., Karachentsev, I. D., Dolphin, A. E., et al. 2008, MNRAS, 384, 1544

Sorce, J. G., Courtois, H. M., \& Tully, R. B. 2012, AJ, 144, 133

Tully, R. B. \& Fisher, J. R. 1977, A\& A, 54, 661 